\title{
OBSERVAÇÕES SÔBRE A ANESTESIA GERAL PELO HIDRATO DE CLORAL NA PRÁTICA DA CIRURGIA VETERINARIA
}

\author{
A. SPALLINI \\ e \\ A. M. PEIXOTO \\ Médico Veterinário \\ Eng. Agrônomo \\ 5a. Cad. da E. S. A. "Luiz de Queiroz"
}

INDICE

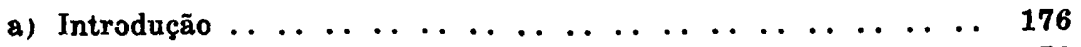
b) Material e Métodos . . . . . . . . . . . . . . . . . . . . . . . 176

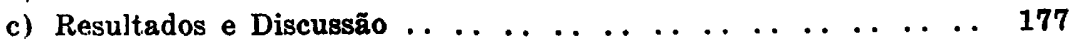
d) Conclusozes $\ldots \ldots \ldots \ldots \ldots$
e) Abstract $\ldots \ldots \ldots \ldots \ldots$
f) Bibliografia . . $\ldots \ldots \ldots \ldots \ldots$ 


\section{a) INTRODUÇÃO}

Esste trabalho tem finalidade essencialmente prática, pois a anestesia geral por via endovenosa com solução de hidrato de cioral é recomendada por diversos autores $(1,2$ e 3$)$ e não constitui, portanto, novidade. Não obstante, as presentes observacões foram realizadas tendo-se em vista a conveniência prática da apiicaçäo do referido agente, graças à facilidade, rapidez e segurança de seu emprêgo, assim como ao seu baixo preço e benignidade.

Realmente, na prática, a simplicidade, segurança e facilidade no uso de qualquer anestésico apresentam importância decisiva. Julgámos mesmo que a difícil aplicação, o alto preço e os riscos, são os principais fatores responsáveis pelo emprêgo quase nulo da anestesia na rotina das intervenções cirúrgicas em veterinária.

O hidrato de cloral é de aplicação simples e rápida, não exigindo conhecimentos especializados; seus efeitos são eficazes, inócuos, suficientemente duradouros, de modo a evitar acidentes na intervenção, protegendo assim o operador e poupando sofrimento ao paciente. Enfim, a sua aplicação é altamente recomendável na prática da cirurgia veterinária nas fazendas de criação, principalmente na castração de equídeos.

\section{b) MATERIAL E MÉTODOS}

Foram feitas aplicações em equídeos e suinos submetidos à castração.

Doses - Para os equídeos, foram usadas de 12-15 g. de hidrato de cloral por $100 \mathrm{k}$ de pêso vivo, em solução de $30 \%$, em água esterijizada a uma temperatura aproximada à do corpo do aninal. Para os suinos, $12-15 \mathrm{~g}$. por $100 \mathrm{k}$. de pêso vivo, em solução de $10-20 \%$.

Vıa de aplicação - Nos equídeos, a solução foi injetada por via enäovenosa, na jugular. Nos suinos, a via geralmente recomendada, a endovenosa auricular, mostrou-se inadequada, em virtude da difícil imobilização e da hemólise frequente do sangue. Foi usado então, com sucesso, o método de Carle e Dewhirst (4), que consiste no seguinte : os porcos são segurados manualmente em decúbito dorsal; usa-se uma seringa de 
vidre de $20 \mathrm{cc}$ e agulha de 1,5 polegadas. A agulha é inserida a $2 \mathrm{~cm}$ da porita da cartilagem cariniforme, em uma linha tirada dêsse ponto à base da orelha e é guiada para dentro, para baixo e para traz, até que sua ponta esteja situada no meio do arco entre as duas primeiras costelas. Nessa área a agulha penetra a veia cava anterior e a injeção pode ser feita com relativa facilidade (fig. 1).

\section{c) RESULTADOS E DISCUSSÃO}

Os quadros A e $B$ indicam raça, sexo, idade e pêso dos animais submetidos à anestesia, assim como os resultados observados nos equídeos e suinos, respectivamente.

Em todos os casos houve relaxamento da verga (fig. 2) dentro de 10-15 minutos após a aplicação do hidrato de cloral. Aliás, êste fenômeno pode ser tomado como indicação segura para 0 início da intervenção.

Os resultados obtidos com as doses empregadas foram bons: perfeito relaxamento muscular (fig. 3), ausência de qualquer contráção e de alterações na temperatura, respiração e pulsação dos pacientes, podendo o operador trabalhar com calma e segurança. O relaxamento dos testículos e orgãos anexos, facilita grandemente a castração dos equídeos, pois, impede a retraçâo que se verifica sempre em animais não anestesiados.

Em geral, a insensibilidade se manifestou entre 5 e $10 \mathrm{mi}-$ nutos após a injeção do anestésico (fig. 4).

Os primeiros sintomas foram: base das orelhas e extremidades frias. Após 15 minutos, observou-se a dilatação das pupilas.

Nas doses fracas houve necessidade de contenção, pois os animais reagiram, embora fracamente, verificando-se contraçñes musculares durante a operação. Isto, nas doses abaixo de $12 \mathrm{~g}$ por $100 \mathrm{k}$. de pêso vivo.

Nas doses boas e fortes, a contenção foi desnecessária (fig. 5) ; nas últimas, porém, a duração da anestesia foi muito prolongada. Em ambas, o relaxamento muscular obtido foi completo.

Ém nenhum dos casos foi notado qualquer alteração sensível ra pulsação, temperatura ou respiração dos animais.

Ac contrário do que afirmam RODER e BERGE (5) a dose empregada permite uma narcose profunda sem ação demasiadamente prolongada do anestésico, no caso dos equídeos. Oferece, também, grande margem de segurança, pois a dose nociva é de 
$37 \mathrm{~g}$. por $100 \mathrm{k}$. de pêso vivo segundo ALFONSO (3), para o cavalo

O emprêgo do hidrato de cloral na anestesia geral por via endovenosa, além das vantagens apontadas, é muito conveniente devido ao custo insignificante : a dose para um cavalo custa de $\operatorname{Cr} \$ 2,00$ a $\operatorname{Cr} \$ 3,00$ e para um suino, de $\operatorname{Cr} \$ 0,10$ a $\operatorname{Cr} \$ 0,30$.

Verificou-se apenas um acidente na aplicação do hidrato de cloral. Tratava-se de um suino gordo $(120 \mathrm{k})$ cuja dose foi calculada de acôrdo com o seu pêso vivo, na base de $20 \mathrm{~g}$. para $100 \mathrm{k}$. A morte ocorreu logo após a injeção.

Aconselhamos, portanto, no caso dos suinos gordos, que o cálculo da dose a empregar seja feito de outra maneira, subtraindo-se do pêso vivo, o pêso do toucinho. Em outras palavras, o touciriho onera o pêso do animal e acarreta uma dose letal, quando esta é baseada diretamente no pêso vivo. $O$ desconto deve ser de aproximadamente $40 \%$, para porcos acima de $100 \mathrm{k}$. de pêso vivo (6).

\section{d) CONCLUSOES}

O presente trabalho permite as seguintes conclusões :

1) O hidrato de cloral, por sua facilidade de aplicação, baixo preço e benignidade, constitui um bom anestésico para equídeos $€$ suinos, na prática da cirurgia veterinária.

2) As doses mais recomendáveis para equídeos e suinos são de $12-13$ g. por $100 \mathrm{k}$. de pêso vivo, em solução de 30 e $20 \%$ em água destilada, respectivamente.

3) O anestésico foi administrado por via endovenosa com bon's resultados; nos equídeos, as injeções foram feitas na jugular; nos suinos, na veia cava anterior, segundo o método de Carle e Dewhirst, pois, a endovenosa auricular mostrou-se impraticável.

4) Os quadros A e B esclarecem os resultados obtidos, que podem ser considerados bons.

5) As doses empregadas permitiram narcose profunda, sem duraçào exagerada e sem perigo para a vida dos animais, graças à grande margem de segurança. 
QUADRO A - EQUÍDEOS

\begin{tabular}{|c|c|c|c|c|c|c|c|c|}
\hline N. & Espécie & Raça & Sexo & $\begin{array}{l}\text { Idade } \\
\text { (anos) }\end{array}$ & $\begin{array}{l}\text { Pêso } \\
\text { (K) }\end{array}$ & Dose & $\begin{array}{l}\text { Duração da } \\
\text { anest. }\end{array}$ & Observação \\
\hline $\begin{array}{l}1 \\
2 \\
3 \\
4 \\
5 \\
6 \\
7 \\
8\end{array}$ & $\begin{array}{l}\text { Equina } \\
\text { Muar } \\
\text { Muar } \\
\text { Muar } \\
\text { Equina } \\
\text { Muar } \\
\text { Muar } \\
\text { Muar }\end{array}$ & $\begin{array}{l}\text { Indeterm. } \\
\text { Indeterm. } \\
\text { Indeterm. } \\
\text { Indeterm. } \\
\text { Indeterm. } \\
\text { Indeterm. } \\
\text { Indeterm. } \\
\text { Indeterm. }\end{array}$ & $\begin{array}{l}\text { Masc. } \\
\text { Masc. } \\
\text { Masc. } \\
\text { Masc. } \\
\text { Masc. } \\
\text { Masc. } \\
\text { Masc. } \\
\text { Masc. }\end{array}$ & $\begin{array}{ll}3 & \\
3 & \\
3 & 1 / 2 \\
3 & 1 / 2 \\
3 & \\
3 & \\
2 & 1 / 2 \\
3 & \end{array}$ & $\begin{array}{l}330 \\
280 \\
200 \\
200 \\
260 \\
220 \\
270 \\
280\end{array}$ & $\begin{array}{l}12 \text { g./100 k. P.V. Sol. } 30 \% \\
15 \text { g./100 k. P.V. Sol. } 30 \% \\
12 \text { g./100 k. P.V. Sol. } 30 \% \\
12 \text { g./100 k. P.V. Sol. } 30 \% \\
12 \text { g./100 k. P.V. Sol. } 30 \% \\
12 \text { g./100 k. P.V. Sol. } 30 \% \\
12 \text { g./100 k. P.V. Sol. } 30 \% \\
15 \text { g./100 k. P.V. Sol. } 30 \%\end{array}$ & $\begin{array}{l}60 \text { minutos } \\
60 \text { minutos } \\
30 \text { minutos } \\
30 \text { minutos } \\
60 \text { minutos } \\
45 \text { minutos } \\
90 \text { minutos } \\
90 \text { minutos }\end{array}$ & $\begin{array}{l}\text { bôa } \\
\text { bôa } \\
\text { bôa } \\
\text { bôa } \\
\text { bôa } \\
\text { bôa } \\
\text { bôa } \\
\text { bôa }\end{array}$ \\
\hline
\end{tabular}

QUADRO B - SUINOS

\begin{tabular}{|c|c|c|c|c|c|c|c|c|c|c|c|c|c|}
\hline \multirow[t]{2}{*}{ N. } & \multirow[t]{2}{*}{ Raça } & \multirow[t]{2}{*}{ Sexo } & \multirow[t]{2}{*}{$\begin{array}{l}\text { Idade } \\
\text { (meses) }\end{array}$} & \multirow[t]{2}{*}{$\begin{array}{l}\text { Pêso } \\
(\mathrm{K})\end{array}$} & \multirow[t]{2}{*}{ Dose } & \multicolumn{2}{|c|}{$\begin{array}{l}\text { Pulsação por } \\
\text { minuto }\end{array}$} & \multicolumn{2}{|c|}{$\begin{array}{c}\text { Temperatura } \\
\text { (Graus C) }\end{array}$} & \multicolumn{2}{|c|}{$\begin{array}{l}\text { Respiração } \\
\text { Movs. por minuto }\end{array}$} & \multirow{2}{*}{$\begin{array}{c}\text { Duração da } \\
\text { Anestesia }\end{array}$} & \multirow[t]{2}{*}{ Observações } \\
\hline & & & & & & In. & Fin. & In. & Fin. & In. & Fin. & & \\
\hline 1 & $\begin{array}{l}\text { Duroc- } \\
\text { Jersey }\end{array}$ & Masc. & 3 & 10,5 & $12 \mathrm{~g} / 100 \mathrm{k}$. PV. Sol. $10 \%$ & 160 & 160 & 39.8 & 39.6 & 28 & 28 & 40 minutos & $\begin{array}{l}\text { Fraca. O animal reagiu fra- } \\
\text { camente à castração }\end{array}$ \\
\hline $\begin{array}{l}2 \\
3 \\
4\end{array}$ & $\begin{array}{l}\text { Nilo } \\
\text { Hampsh. } \\
\text { Duroc- }\end{array}$ & $\begin{array}{l}\text { Masc. } \\
\text { Masc. }\end{array}$ & $\begin{array}{l}3 \\
3\end{array}$ & $\begin{array}{l}12,0 \\
10,0\end{array}$ & $\begin{array}{l}12 \mathrm{~g} / 100 \mathrm{k} . \text { PV. Sol. } 20 \% \\
12 \mathrm{~g} / 100 \mathrm{k} . \text { PV. Sol. } 20 \%\end{array}$ & $\begin{array}{l}155 \\
126\end{array}$ & $\begin{array}{l}155 \\
115\end{array}$ & $\begin{array}{l}39,8 \\
40.4\end{array}$ & $\begin{array}{l}40.0 \\
40.6\end{array}$ & $\begin{array}{l}24 \\
42\end{array}$ & $\begin{array}{l}24 \\
32\end{array}$ & $\begin{array}{l}40 \text { minutos } \\
40 \text { minutos }\end{array}$ & $\begin{array}{l}\text { s Bôa. Reação débil } \\
\text { s Bôa. Reação fraca }\end{array}$ \\
\hline $\begin{array}{l}5 \\
6\end{array}$ & $\begin{array}{l}\text { Jersey } \\
\text { Nilo } \\
\text { Duroc- }\end{array}$ & $\begin{array}{l}\text { Masc. } \\
\text { Fem. }\end{array}$ & $\begin{array}{l}3 \\
3\end{array}$ & $\begin{array}{l}13.0 \\
13.5\end{array}$ & $\begin{array}{l}13 \mathrm{~g} / 100 \mathrm{k} . \text { PV. Sol. } 20 \% \\
14 \mathrm{~g} / 100 \mathrm{k} . \text { PV. Sol. } 20 \%\end{array}$ & $\begin{array}{l}112 \\
133\end{array}$ & $\begin{array}{l}112 \\
150\end{array}$ & $\begin{array}{l}40.6 \\
39.7\end{array}$ & $\begin{array}{l}38.4 \\
39,9\end{array}$ & $\begin{array}{l}36 \\
55\end{array}$ & $\begin{array}{l}24 \\
48\end{array}$ & $\begin{array}{l}40 \text { minutos } \\
60 \text { minutos }\end{array}$ & \begin{tabular}{l|l} 
s & Bôa. Não houve reação \\
s & Bôa. Não houve reação
\end{tabular} \\
\hline 7 & $\begin{array}{l}\text { Jersey } \\
\text { Duroc- }\end{array}$ & Masc. & 3 & 12,2 & $15 \mathrm{~g} / 100 \mathrm{k}$. PV. Sol. $20 \%$ & 120 & 104 & 38.0 & 38.6 & 40 & 40 & 95 minutos & s $\begin{array}{l}\text { Muito forte. Não houve } \\
\text { reação }\end{array}$ \\
\hline & Jersey & Masc. & 3 & 17,4 & $15 \mathrm{~g} / 100 \mathrm{k}$. PV. Sol. 20\% & 104 & 128 & 38.7 & 38.7 & 28 & 24 & 95 minutos & s $\left.\right|_{\text {reação }} ^{\text {Muito forte. Não houve }}$ \\
\hline $\begin{array}{l}8 \\
9\end{array}$ & $\begin{array}{l}\text { Hampsh. } \\
\text { Duroc- }\end{array}$ & Masc. & 3 & 18,0 & 14,5 g/100 k. PV. Sol. $20 \%$ & 120 & 130 & 38.9 & 38.9 & 40 & 50 & 65 minutos & sorte. Não houve reação \\
\hline $\begin{array}{l}10 \\
11\end{array}$ & $\begin{array}{l}\text { Jersey } \\
\text { Hampsh. } \\
\text { Hampsh. }\end{array}$ & $\begin{array}{l}\text { Fem. } \\
\text { Masc. } \\
\text { Fem. }\end{array}$ & $\begin{array}{l}4 \\
4 \\
4\end{array}$ & $\begin{array}{l}11.0 \\
19.0 \\
120,0\end{array}$ & $\begin{array}{l}14 \mathrm{~g} / 100 \mathrm{k} . \text { PV. Sol. } 20 \% \\
15 \mathrm{~g} / 100 \mathrm{k} . \text { PV. Sol. } 20 \% \\
20 \mathrm{~g} / 100 \mathrm{k} . \text { PV. Sol. } 48 \%\end{array}$ & $\begin{array}{r}88 \\
168 \\
-\end{array}$ & $\begin{array}{r}96 \\
140 \\
-\end{array}$ & $\begin{array}{c}38.9 \\
38.8 \\
-\end{array}$ & $\begin{array}{c}38.7 \\
39.6 \\
-\end{array}$ & $\begin{array}{l}40 \\
52 \\
-\end{array}$ & $\begin{array}{l}30 \\
44 \\
-\end{array}$ & $\left|\begin{array}{l}80 \text { minutos } \\
50 \text { minutos } \\
-\quad-\end{array}\right|$ & $\begin{array}{l}\text { s Forte. Não houve reação } \\
\text { s Bôa. Não houve reação } \\
\text { Morte rápida }\end{array}$ \\
\hline
\end{tabular}


6) No caso de suinos gordos, para o cálculo da dose a ser empregada, deve ser feito um desconto de aproximadamente $40 \%$ no pêso vivo.

\section{e) ABSTRACT}

In this paper the A. A. report the observations about the general anesthesia by chloral hydrate on the veterinary surgery. The observations were made on emasculation practices of horses, mules and hogs. It was possible to establish the following conclusions :

1) The choral hydrate presents low cost, it harmless, and is of easy application.

2) The more recommendable dosis for equine and swine were : $12-13 \mathrm{~g}$ per $100 \mathrm{k}$ of body weight, in destilled water solution at 30 and $20 \%$, respectively.

3) The anaethestic was injected by intravenous way with good results; in horses and mules the applications were made in the jugular; in swine, in the anterior vena cava, as was described by Carle and Dewhirst, because it was impracticable in the ear vein.

4) The dosis applied produced deep narcosis not lasting to long and with no danger to the animal's life.

5) In the case of fattening hogs, it must be made a discount of about $40 \%$ on the body weight, to calculate dosis to be employeä.

6) The tables $A$ and $B$ show the results, that may be considered as good.

\section{f) BIBLIOGRAFIA}

1. ADAMI, E. - Manuale di Farmacologia e Farmocoterapia Veterinaria. II Edizione. Instituto Editoriale Cisalpino. Milano. Varese. 1948. 
2. MOLLEREAU, H., PORCHER, C. et NICOLAS, E. - VadeMecum du Vétérinaire. Huitième Edition. Vigot Fréres, Editeurs. Paris. 1949.

3. ALFONSO, G. C. - Tratado de Operaciones en Veterinaria. Segunda Edicion. Imprenta Biosca. Madrid. 1948.

4. COFFIN, D. L. - Manual of Veterinary Clinical Pathology. College Offset Press. Philadelphia. 1944.

5. RODER, O. e BERGE, E. - Técnica Operatória Veterinaria. Trad. de la Quinta Edición Alemana. Editorial Labor, S. A. Barcelona. 1947.

6. EGAN̄A, C. S. - Enciclopedia de la Carne. Espasa-Calpe, S. A. Madrid. 1948. 


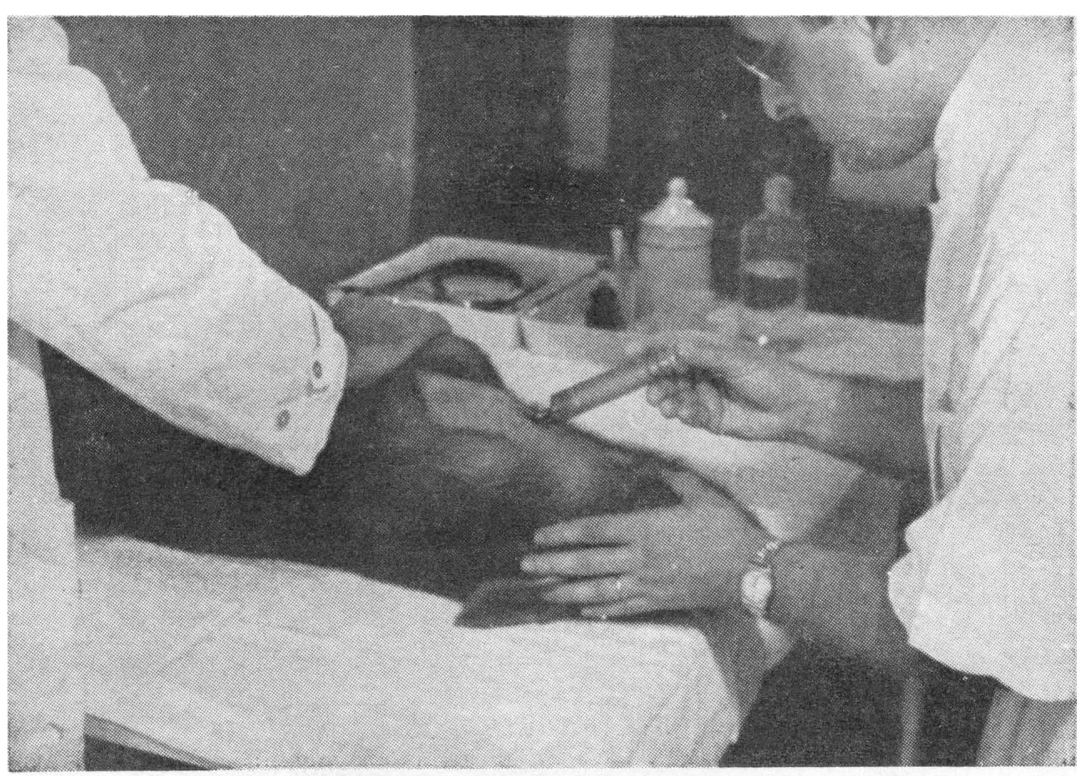

Fig. 1

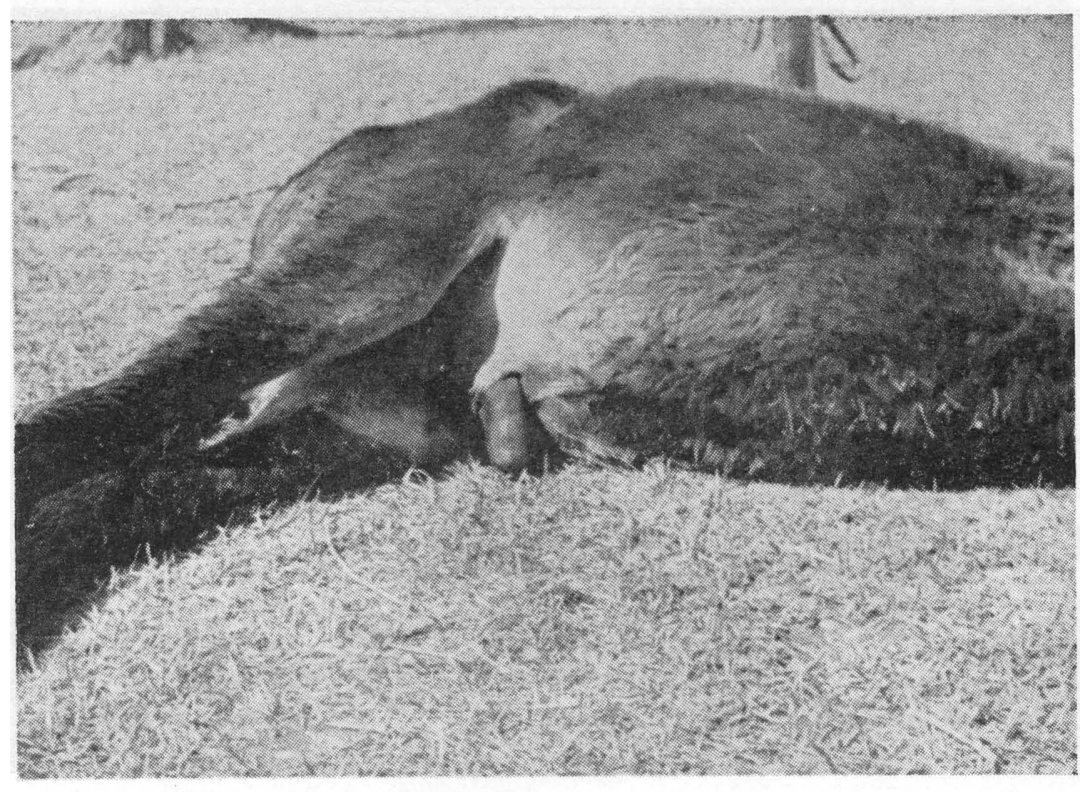

Fig. 2 


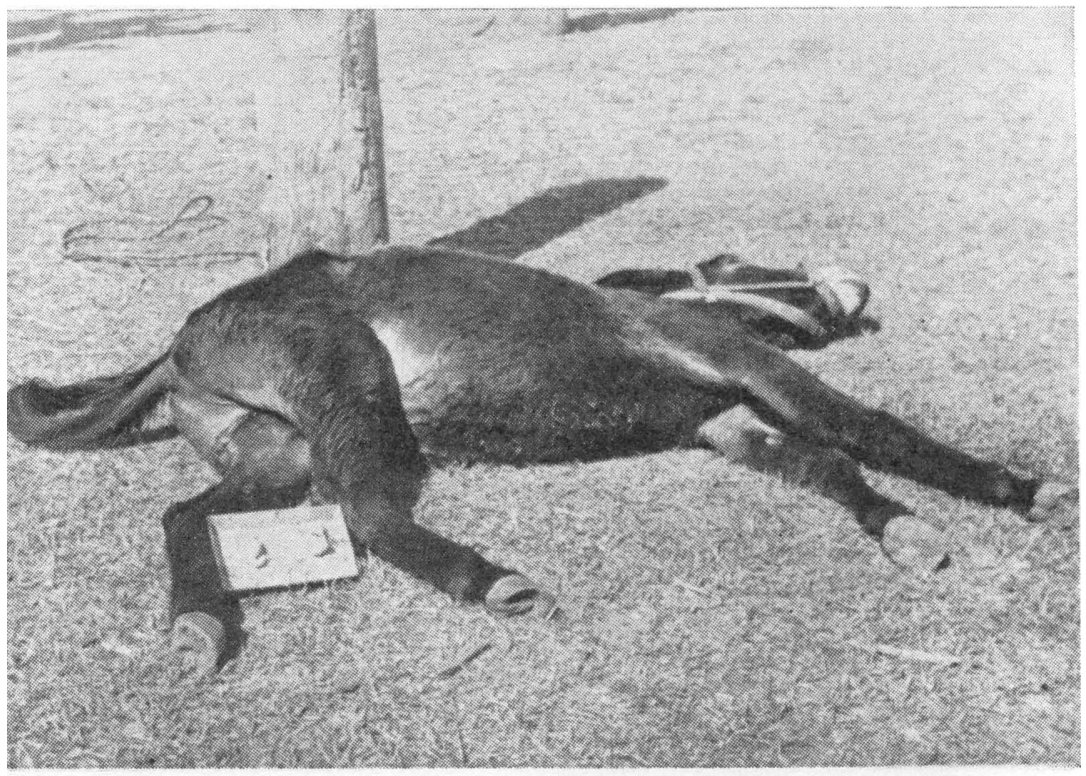

Fig. 3

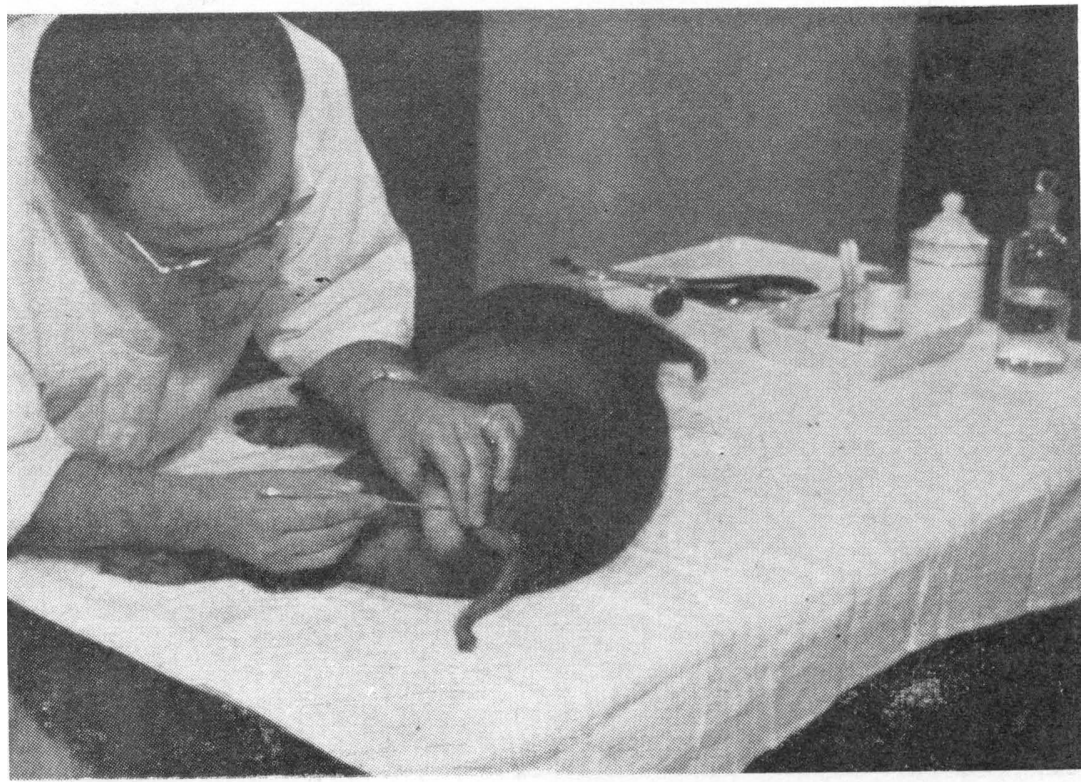

Fig. 4 


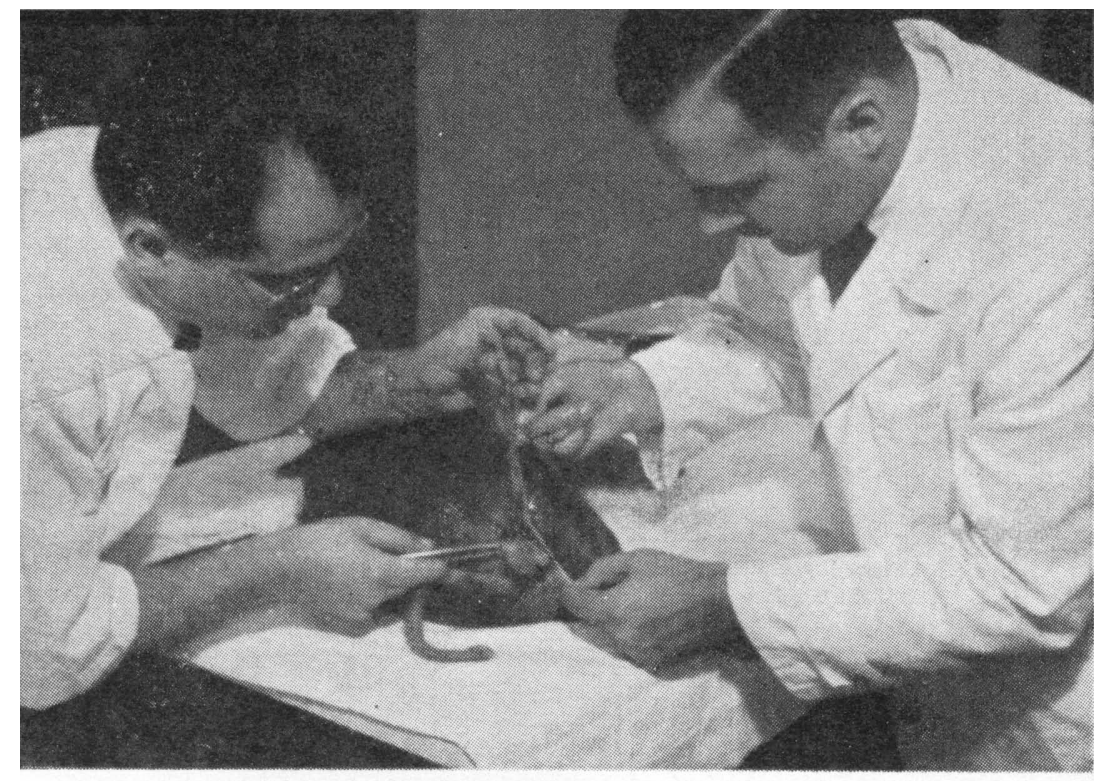

Fig. 5 